Historic, Archive Document

Do not assume content reflects current scientific knowledge, policies, or practices. 



\section{Choice Selections}

- FOR

\section{Spring Planting}

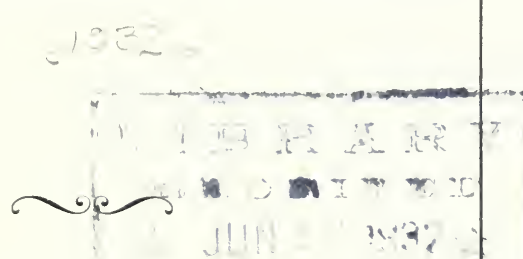

U. 8. Deperment a A gacuitre.

WE believe this list of broad-teaf evergreens and other plants contains items of outstanding interest for 1932 gardening, and we also believe that material of this high cuality will not be available again for many years at these low prices.

:: $\quad$ :

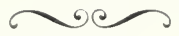

GREENE'S EVERGREEN NURSERY

WHITNEY E. GREENE

DOVER, MASSACHUSETTS

Specializing in Woodland Plantings

TELEPHONE： DOVER - 186 


\section{BROAD-LEAF EVERGREENS}

RHODODENDRON MAXIMUM - Thick, bushy clumps, $1-2 \mathrm{ft}$. and $2-3 \mathrm{ft}$., at $\$ 1.50$ and $\$ 2.50$ each. Massive clumps, well budded, 3-4 ft. $4-5 \mathrm{ft}$. and 5-6 ft., at $\$ 3.50, \$ 5.00$ and $\$ 7.50$ each.

RHODODENDRON CATA IVBIENSE - Thick, bushy clumps, $2-2 \frac{1}{2} \mathrm{ft}$., budded, at $\$ 2.50$ ea. Massive clumps, heavily budded, $3-4 \mathrm{ft}$. and 4-5 ft., selected, at $\$ 3.50$ and $\$ 5.00$ each.

RHODODENDRON CAROLINIANUM - Well developed budded clumps, $2-2^{1} \frac{1}{2} \mathrm{ft}$., at $\$ 3.50$ ea.

HYBRID RHODODENDRON SEEDLINGS-Pink, white and red-not named, sizes up to $2 \mathrm{ft}$., some budded, $75 \mathrm{c}$ to $\$ 2.75$ each.

NAMED HYBRID RHODODENDRONS--Well developed plants from grafts, several buds, 2$21 \% 2 \mathrm{ft}$., at $\$ 4.75$ each.

KAIMIA LATIFOLIA-Mountain laurel, unusually thick clumps of over 15 main branches, about $20^{\prime \prime}-24^{\prime \prime}$ high, at $\$ 2.50$ each. Also 2-3 $\mathrm{ft} ., 3-4 \mathrm{ft}$. and $4-5 \mathrm{ft}$. clumps for $\$ 3.50, \$ 5.00$ and $\$ 7.50$ each.

PIERIS FLORIBUNDA - Mountain andromeda, very compact spreading specimens, heavily budded, 18-24" at $\$ 2.50$ each.

PIERIS JAPONICA-Japanese andromeda. Beautiful specimens, 15-18", well budded, at $\$ 2.75$ ea. Also 2-3 ft. and $3-4 \mathrm{ft}$., specimens at $\$ 5.00$ and $\$ 7.50$ each.

ILEX GLABRA - Inkberry. Unusually dense shapely clumps, $3-4 \mathrm{ft}$. at $\$ 4.50$ each. Ideal for background or hedge. Also $1 \frac{1 \% 2}{1}-2 \mathrm{ft}$. and $2-3 \mathrm{ft}$. at $\$ 2.50$ and $\$ 3.50$ each.

LECCOTHOE CATESBAEI-Drooping leucothoe, 18-24" spread, well budded, at $\$ 1.95$ each.

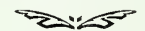

\section{AZALEAS}

AZALEA CALENDULACEA-Flame azalea, large budded clumps, $2-2^{1} \ldots \mathrm{ft}$., at $\$ 1.95$ ea., $3-4 \mathrm{ft}$., at $\$ 3.50$ each.

AZALEA NUDIFLORA - Pinksterbloom. Fully developed, budded clumps, $3-4 \mathrm{ft}$., at $\$ 3.50$.

AZALEA CANESCENS - Piedmont azalea. Well budded, $3-4 \mathrm{ft}$., at $\$ 2.75$ each.

AZALEA VISCOSA - Swamp white azalea, July bloom, 8-10 branch, well shaped, budded clumps, 3-4 ft., at only $\$ 1.95$ each.

AZALEA MOLLIS-Chinese azalea, 15-18" specimen plants, budded, at $\$ 2.75$ each.

AZALEA HINODEGIPI-Bright red. 12-15" budded specimens, at $\$ 2.75$ each. 


\section{Washington Bicentennial Tree Label or Plaque}

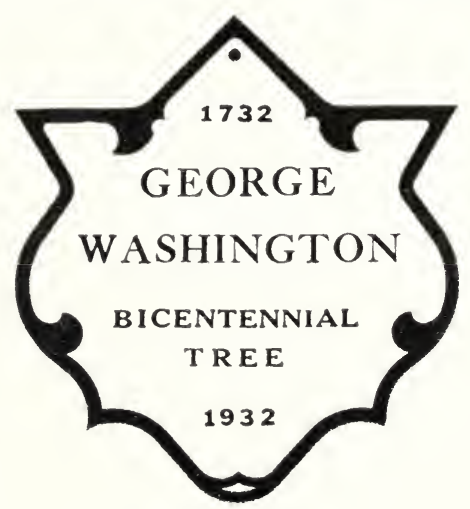

Copyrighted 1932, By W. E. G.

This year the Government is encouraging the planting of ten million trees to commemorate the 20oth Anniversary of George Washington's Birthday. Use this beautifully designed, indestructible, bronze plaque to mark your memorial trees. They are inexpensive and completely equipped for attaching.

SMALL SIZE: I very durable stamping of sheet bronze with attractive border as illustrated. '2 inches high. equipped with copper wire for attaching. POSTPAID 15c. EA.

INTERMEDIATE SIZE: A heary solid statue, bronze casting, $21 / 2$ " high and 1 " thick. Letters ant border are nicely entbossed against stippled background. Complete with copper attachment. POSTPAJD 50c. EA.

LARGE SIZE: I heavy solid statue-bronze casting with raised letters and borker against stippled background. 5" high by 11.2" wide. This beautiful plaque may be had equipped with two bushings and brass serews to be attached directly to tree, or with 12" metal rod to thurst in ground.

POSTPAID $\$ 2.75$ EA.

GREENE'S EVERGREEN NURSERY, - DOVER, MASSACHUSETTS 

BUXUS SEMPERVIRENS - Bush box 4-6" and 6-8" for edging, at $15 \mathrm{c}$ and $25 \mathrm{c}$ ea. 21-24" specimens, at $\$ 3.50$ each.

BUXUS SUFFRUTICOSA-Edging sizes as above, or specimens up to $10 \mathrm{ft}$, from $\$ 50.00$ to $\$ 800.00$ each. Photographs any size on request.

WHITE FLOWERING DOGWOODS - Unusually choice shapely clumps, 6-10 branches, each. 1" caliper, 7-9 ft. high, 50-100 buds (value $\$ 20.00$ to $\$ 25.00$ ea.) only $\$ 9.00$. Smaller clumps, 3-5 branches, $5-7 \mathrm{ft}$. high, at $\$ 5.00$ ea.

Single stem specimens, 11/4" caliper and rough bark at base. $\$ 1.95$ each.

PINK FLOWERING DOGWOOIS-Grafted stock, perfectly shaped, $2^{1 / 2}-3 \mathrm{ft}$., at $\$ 3.00$ ea. $3^{1 / 2-4}$ $\mathrm{ft.}$ at $\$ 4.75$ ea., and $4^{1}-2-5 \mathrm{ft} ., \$ 8.50$

MAGNOLIA SOULANGEANA - 3ft. at $\$ 8.50$ each.

PYRACANTHA COCCINEA LEIANI)I - Leland's fire thorn, $3^{1}-4 \mathrm{ft}$., at $\$ 6.50$ each.

PAUL'S SCARIET HAWTHORN - 5-6 ft., \$3.25; and $6-7 \mathrm{ft} ., \$ 4.50$ each.

VIBURNLI CARIESII - These are excellent plants and very desirable. 15-18" at $\$ 2.50$ and $2-2{ }^{2}: 2 \mathrm{ft}$. at $\$ 3.50$ each.

DAPHNE MEZERETM - 15-18" specimens, $\$ 2.50$.

DAPHNE CNEORUM-12-15" at $\$ 1.25$ ca. and $8-10 "$ at only 90 ce each.

JAPANESE FIOOWERING CRABS-(Malus) - - 5-(; ft., in these varieties: Parkmani, Theifera, Bechtels, Zumi callicarpa, Eleyi and Niedzwetzkyana, all at $\$ 3.50$ ea. These are fine for group plantings.

JAPANESE FI.ONERING CHERRIES-(Prunus) Prunus Triloba $-33^{1 / 2-4} \mathrm{ft}$. at $\$ 1.50$ each.

Prunus Pissardi $-6-7 \mathrm{ft}$. at $\$ 2.75$ each

Prunus Newport Plum - 5-6 ft. at $\$ 2.50$ each.

Prunus Subhirtella Rosea Pendula - (Weep)ing Japanese Cherry)-4-5 ft., at $\$ 4.50$ ea.

HYBRII) LIIACS in the following ten varieties: Charles X, Congo, Marie Le Gray, Souv. Ludwig Spaeth, Belle de Nancy, Dr. Nasters, Michael Buchner, Mme. Lemoine, Pres. Grevy, and Ville de Troys. All $3-4 \mathrm{ft}$ from grafts, $\$ 1.00$ ea., or $\$ 9.00$ any ten.

COTONEASTER HORIZONTALIS - 6" pots, 12$15 "$ spread at $\$ 1.50$ each.

COTONEASTER IDELSIANA - 2-3 ft., at $\$ 2.50$ each. 


\section{OTHER ITEMS FOR WOODLAND DEVELOPMENT}

AMELANCHIER CANADENSIS - Shad or Juneberry, $3-4 \mathrm{ft}$. at $90 \mathrm{c}$ ea., $4-5 \mathrm{ft}$. at $\$ 1.50$ ea., $5-6$ ft. specimens at $\$ 2.50$ each.

ILEX VERTICILLATA - Winterberry or black alder. $3-4 \mathrm{ft}$. at $90 \mathrm{c}$ ea. and $4-5 \mathrm{ft}$. at $\$ 1.50$ ea.

HAMAMELIS VIRGINIANA - Witch hazel, 5-6 ft. $90 \mathrm{c}$.

BERBERIS VULGARIS - European barberry. Very attractive foliage and berries. $3-5 \mathrm{ft}$. clumps, $\$ 2.50$ each.

ARONIA ARBUTIFOLIA - Red chokeberry, also very desirable and attractive. $3-3 \frac{1}{2} \mathrm{ft}$. nursery grown, at $\$ 1.00$ each.

VIBURNUM CASSINOIDES-IVitherod. Unusually well branched shapely clumps, 3-5 ft. high, at only $\$ 2.50$ each.

VACCINIUM CORYMBOSUM - High bush blueberry. Young healthy clumps, 2-3 ft. at $\$ 1.75$.

TSUGA CANADENSIS - Hemlock. 2-3 ft., 4-5 ft. and $6-8$ ft., $\$ 2.50, \$ 5.00$ and $\$ 7.50$ each.

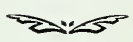

\section{SUGGESTIONS FOR WASHINGTON BICENTENNIAL MEMORIAL TREES}

CERCIS CANADENSIS - Judas tree or Ped-bud. purple flowers before leaves.

OXYDENDRON ARBOREUM-Sourwood. A very attractive tree with buds and flowers similar to andromeda. Remarkable Fall coloring.

PERSIMION - Attractive fruit.

SYCAMORE - Fast growing large tree.

KOELREUTARIA PANICULATA - Golden Rain tree or Varnish tree. Beautiful orange-yellow flowers, large tree. $\$ 2.50$ each.

LABURNUM VULGARE-Golden Chain tree. An ideal lawn specimen, flowers yellow in panicles.

SORBUS AUCUPARIA-European Mountain Ash, attractive foliage and berries, $\$ 2.50$ each.

All above trees are $6-\delta \mathrm{ft}$. and at $\$ 1.75$ each, except when noted.

Also a large assortment of evergreens, deciduous trees and shrubs, perennials, roses, and rock garden plants.

ALL PRICES IN THIS LIST INCLUDE DELIVERING AND PLANTING ON YOUR ESTATE AT YOUR CONVENIENCE. TO DISTANT POINTS THESE PRICES INCLUDE PACKING. $10 \%$ DISCOUNT FOR PAYMENT WTH ORDER. 